\title{
АНАЛИЗ ХИМИЧЕСКОГО ПОТЕНЦИАЛА ДВУМЕРНОГО ЭЛЕКТРОННОГО ГАЗА ПРИ НИЗКИХ ТЕМПЕРАТУРАХ
}

\author{
П.Ж. Байматов ${ }^{1}$, А.Г. Гулямов ${ }^{2}$, Б.Т. Абдулазизов ${ }^{2}$, А.С. Махмудов ${ }^{1}$ \\ ${ }^{1}$ Наманганский государственный университет, 160119, Наманган, Узбекистан \\ ${ }^{2}$ Физико-технический институт АН РУз, 100084, Ташкент, Узбекистан
}

(Получена 13.01.2020)

\begin{abstract}
Икки ўлчамли электронлар газининг кимёвий потенциал $\mu$ ни резонанс нуқтталар атрофида (минизоналар тубида) ва ундан узоқроқ нуқтталарда хусусиятлари қุараб чиқ़илган. Паст температураларда ва минизоналар сони ихтиёрий бўлган шароитларда аналитик формулалар олинган. Температура ортиб боришида, резонанс нуқтталар яқцннида кимёвий потенциални чизикฺли қьонун бўйича камайиши, резонанс нуқ̨талар ораларида эса экспоненциал секин камайиши кўрсатилган.
\end{abstract}

\begin{abstract}
Рассматривается поведение химического потенциала $\mu$ двумерного электронного газа вблизи дна минизоны и вдали от нее. Получены низкотемпературные аналитические формулы в условиях существование произвольного числа минизон. Показано, что с ростом температуры вблизи точек резонанса химический потенциал уменьшается линейно, а вдали от нее экспоненциально медленно.
\end{abstract}

The behavior of the chemical potential $\mu$ of a two-dimensional electron gas near the bottom of the miniband and far from it is considered. Low-temperature analytical formulas are obtained under the conditions of the existence of an arbitrary number of minibands. It has been shown that with increasing temperature near the resonance point the chemical potential decreases linearly, and away from it exponentially slowly.

\section{I. ВВЕДЕНИЕ}

Изучение свойств электронного газа в квантовых ямах полупроводниковых гетероструктур вызывает как фундаментальный, так и прикладной интерес. Размерное квантование энергии носителей приводит к проявлению ряда интересных низкоразмерных эффектов [1-14]. В глубоких квантовых ямах могут существовать несколько минизон [15-17].

В настоящее время хорошо известно, что в приближении одной минизоны и при низких температурах химический потенциал квазидвумерного электронного газа уменьшается экспоненциально медленно [8, 9]. Зависимость $\mu(T)$ в присутствии нескольких минизон изучена в работе [14], однако результаты получены только численным методом. Так как почти все термодинамические величины и кинетические коэффициенты непосредственно связаны с химическим потенциалом газа, то ясно, что определение функции $\mu(T)$ является актуальной задачей.

В данной работе для зависимости $\mu(T)$ получены низкотемпературные аналитические формулы. Полученные результаты показали, что с ростом температуры вблизи точек резонанса химический потенциал уменьшается линейно, а в середине резонансных точек - экспоненциально медленно. 


\section{II. ОСНОВНЫЕ СООТНОШЕНИЯ}

Рассматривается идеальный электронный газ с простым параболическим спектром в одиночной квантовой яме. При этом не учитывается тепловой переход электронов из квантовой ямы на локальные уровни и в зону проводимости широкозонного материала. Пренебрегается также влияние валентной зоны. Энергия отсчитывается от дна зоны проводимости массивного полупроводника.

В квантовой яме энергия поперечного движения квантуется, и электронный газ становится квазидвумерным. Дисперсию электрона можно представить в виде [1]

$$
E=\frac{\hbar^{2} k^{2}}{2 m}+E_{n}
$$

где $k^{2}=k_{x}^{2}+k_{y}^{2}, m$ - эффективная масса электрона, $E_{n}$ - энергетические уровни поперечного движения. Нахождение уровней $E_{n}$ является квантовомеханической частью задачи, и мы будем считать их известными. Полная двумерная концентрация равна

$$
n_{s}=g_{0} \sum_{n=1}^{M} \int_{E_{n}}^{\infty} f(E) d E=g_{0} T \sum_{n=1}^{M} \ln \left(1+\exp \left[\frac{\mu-E_{n}}{T}\right]\right),
$$

где $g_{0}=m / \pi \hbar^{2}, M$ - число минизон в данной квантовой яме, через $T$ обозначена величина $k_{B} T, k_{B}$ - постоянная Больцмана, $f(E)$ - функция распределения ФермиДирака. Решая уравнение (2), можно определить химический потенциал $\mu$ как функцию температуры $T$ и двумерной концентрации $n_{s}$.

При нулевой температуре химический потенциал равен энергии Ферми, т.е. $T=0, \mu=E_{F}$, а функция распределения $f(E)$ становится ступенчатой. Тогда при $T=0$ уравнение (2) принимает вид

$$
n_{s}=g_{0} \sum_{n=1}^{M} \int_{E_{n}}^{E_{F}} d E=g_{0} \sum_{n=1}^{M}\left(E_{F}-E_{n}\right) \Theta\left(E_{F}-E_{n}\right) .
$$

Здесь $\Theta(x)$ - функция Хевисайда. В уравнении (3) слагаемые под знаком суммы должны быть положительными. Отрицательные слагаемые исключаются функц0ией $\Theta(x)$. Из (2) и (3) следует также уравнение

$$
\sum_{n=1}^{M}\left(E_{F}-E_{n}\right) \Theta\left(E_{F}-E_{n}\right)=T \sum_{n=1}^{M} \ln \left(1+\exp \left[\frac{\mu-E_{n}}{T}\right]\right) .
$$

Это уравнение определяет химический потенциал $\mu$ как функцию температуры $T$ и энергии Ферми $E_{F}$. Так как согласно (3) $E_{F}$ однозначно связана с двумерной концентрацией $n_{s}$, то ниже будет исследовано только решение уравнения (4).

\section{III. АНАЛИЗ УРАВНЕНИЯ (4) ПРИ НИЗКИХ ТЕМПЕРАТУРАХ}

1. Сначала рассмотрим ситуацию, когда химический потенциал лежит примерно в середине двух последовательных уровней $N$ и $N+1, E_{N}<\mu<E_{N+1}$ и температура достаточно низка $T<<\mu-E_{N}, E_{N+1}-\mu$. Тогда правую часть уравнения (4) можно свести к виду: 


$$
\sum_{n=1}^{M} T \ln \left(1+\exp \left[\frac{\mu-E_{n}}{T}\right]\right) \approx \sum_{n=1}^{N}\left(\mu-E_{n}\right)+T \ln \left(1+\exp \left[\frac{E_{n}-\mu}{T}\right]\right)+T \ln \left(1+\exp \left[\frac{\mu-E_{n+1}}{T}\right]\right)
$$

В таком представлении второй и третий члены имеют одинаковые степени малости. В рассматриваемом приближении левая часть уравнения (4) равна

$$
\sum_{n=1}^{M}\left(E_{F}-E_{n}\right) \Theta\left(E_{F}-E_{n}\right)=N E_{F}-\sum_{n=1}^{N} E_{n}
$$

Подставляя (5) и (6) в (4), имеем

$$
\mu \approx E_{F}-\frac{T}{N}\left[\ln \left(1+\exp \left[\frac{E_{N}-\mu}{T}\right]\right)+\ln \left(1+\exp \left[\frac{\mu-E_{N+1}}{T}\right]\right)\right] .
$$

Если учесть условие $T<<\mu-E_{N}, E_{N+1}-\mu$, то это уравнение можно упростить дальше. При малых $x$ воспользуемся разложением $\ln (1+x) \approx x$ и из (7) получим

$$
\mu \approx E_{F}-\frac{T}{N}\left(\exp \left[\frac{E_{N}-\mu}{T}\right]+\exp \left[\frac{\mu-E_{N+1}}{T}\right]\right) .
$$

В первом приближении в правой части (8) можно заменить $\mu$ на $E_{F}$. Тогда имеем

$$
\mu \approx E_{F}-\frac{T}{N}\left(\exp \left[\frac{E_{N}-E_{F}}{T}\right]+\exp \left[\frac{E_{F}-E_{N+1}}{T}\right]\right) .
$$

Из (9) видно, что при низких температурах уменьшение химического потенциала двумерного газа экспоненциально мало. Эта поправка обратно пропорциональна номеру подзоны $N$. Допущенное условие $T<<\mu-E_{N}, E_{N+1}-\mu$ не позволяет применить формулу (9) в случаях, когда энергия Ферми находится близко к уровням $E_{N}, E_{N+1}$.

2. Пусть энергия Ферми лежит вблизи $N$-го уровня (дна $N$-ой минизоны) и температура достаточно низка $T \sim T_{N}>0, \quad\left|\mu-E_{N}\right|<<T<<\mu-E_{N-1}, E_{N+1}-\mu$. Здесь $T_{N}$ есть температура, при которой химический потенциал пересекает энергетический уровень $E_{N}$. Тогда правую часть (4) можно упростить:

$$
\sum_{n=1}^{M} \ln \left(1+\exp \left[\frac{\mu-E_{N}}{T}\right]\right) \approx \frac{1}{T}(N-1) \mu-\frac{1}{T} \sum_{n=1}^{N-1} E_{n}+\ln \left(1+\exp \left[\frac{\mu-E_{N}}{T}\right]\right) .
$$

Поскольку $\left|\mu-E_{N}\right|<<T$, то можно воспользоваться следующими разложениями

$$
\exp \left(\frac{\mu-E_{N}}{T}\right) \approx 1+\frac{\mu-E_{N}}{T}, \quad \ln \left(1+\frac{\mu-E_{N}}{2 T}\right) \approx \frac{\mu-E_{N}}{2 T} .
$$

Тогда после некоторых упрощений в (10) имеем

$$
\sum_{n=1}^{M} \ln \left(1+\exp \left[\frac{\mu-E_{n}}{T}\right]\right) \approx \frac{\mu}{T}\left(N-\frac{1}{2}\right)-\frac{E_{N}}{2 T}-\frac{1}{T} \sum_{n=1}^{N-1} E_{n}+\ln 2 .
$$

В рассматриваемом приближении левая часть уравнения (4) равна 


$$
\sum_{n=1}^{M}\left(E_{F}-E_{n}\right) \Theta\left(E_{F}-E_{n}\right)=(N-1) E_{F}-\sum_{n=1}^{N-1} E_{n}+\left(E_{F}-E_{N}\right) \Theta\left(E_{F}-E_{N}\right)
$$

Подставляя (11) и (12) в (4), имеем

$$
\mu=\frac{(N-1) E_{F}+\left(E_{F}-E_{N}\right) \Theta\left(E_{F}-E_{N}\right)+\frac{E_{N}}{2}-T \ln 2}{N-(1 / 2)} .
$$

В случае $E_{F}>E_{N}(13)$ упрощается

$$
\mu=\frac{N E_{F}-\left(E_{N} / 2\right)-T \ln 2}{N-(1 / 2)},
$$

а в случае $E_{F}<E_{N}$

$$
\mu=\frac{(N-1) E_{F}+\left(E_{N} / 2\right)-T \ln 2}{N-(1 / 2)} .
$$

Отсюда видно, что с ростом температуры вблизи $N$-го уровня химический потенциал уменьшается линейно. При точном резонансе $\mu=E_{N}, T=T_{N}, E_{F}>E_{N}$ из (13) получаем

$$
T_{N}=N\left(E_{F}-E_{N}\right) / \ln 2 \text {. }
$$

Таким образом, если уровень Ферми достаточно близок к $N$-му уровню, то допущенные условия $T \sim T_{N}>0, \quad\left|\mu-E_{N}\right|<<T<<\mu-E_{N-1}, E_{N+1}-\mu$ хорошо выполняются. При выводе формул (13)-(16) уровнями $n>N$ мы пренебрегали, а слагаемые с номерами $n<N$ суммировали приближенно согласно условию $T<<\mu-E_{N-1}, E_{N+1}-\mu$. В противном случае зависимость $\mu(T)$ и температуру $T_{N}$ (при котором осуществляется резонанс $\mu=E_{N}$ ) необходимо определить из (4) численным путем.

3. Рассмотрим случай, когда энергия Ферми точно равна $N$-му энергетическому уровню: $E_{F}=E_{N}$. С ростом температуры химический потенциал смещается вниз. Полагая $E_{F}=E_{N}$, из (13) можно получить следующую формулу

$$
\mu \approx E_{N}-\frac{T \ln 2}{N-(1 / 2)} .
$$

Если исходить из (4), то при выполнении условия $T<<\mu-E_{N-1}, E_{N+1}-\mu$, положив $E_{F}=E_{N}$, приходим к следующему уравнению

$$
(N-1) \mu+T \ln \left(1+\exp \left[\frac{\mu-E_{N}}{T}\right]\right)=(N-1) E_{N}, \quad N>1 .
$$

Введя обозначение $x=\exp \left[\left(E_{N}-\mu\right) / T\right]$, ур-е (18) можно привести к виду

$$
x+1=x^{N}, \quad N>1 .
$$

Тогда находим, что химический потенциал меняется с температурой по закону

$$
\mu=E_{N}-T \ln x .
$$

При выводе (17) использованы разложения экспоненты и логарифма в ряд, в то время как при выводе формулы (20) отказались от этого разложения. Для их сравнения ниже приведены расчетные данные для $\ln x$ и $\ln (2) /(N-1 / 2)$ : 


\begin{tabular}{c|c|c}
\hline$N$ & $\ln x$ & $\ln (2) /(N-(1 / 2))$ \\
\hline 2 & 0.481 & 0.462 \\
3 & 0.281 & 0.277 \\
4 & 0.199 & 0.198 \\
5 & 0.155 & 0.154 \\
6 & 0.126 & 0.126 \\
\hline
\end{tabular}

Отсюда видно, что при $N=2$ их разница составляет около одного процента, а с ростом $N$ быстро уменьшается.

\section{IV. СРАВНЕНИЕ С ЧИСЛЕННЫМИ РЕЗУЛЬТАТАМИ И ОБСУЖДЕНИЕ \\ РЕЗУЛЬТАТОВ}

Для конкретных квантовых ям гетероструктуры энергетические уровни $E_{N}$ определяются квантовомеханически. Для численного решения уравнения (4) используем простой спектр $E_{n}=E_{1} n^{2}, n=1,2,3 \ldots$, с заданным $E_{1}$.

На рис. 1 представлено сравнение зависимостей $\mu(T)$, полученных по аналитической формуле (9), с результатами численного решения уравнения (4) для разных значений энергии Ферми. При расчетах использованы следующие параметры спектра: $E_{1}=0.005 \mathrm{eV}, E_{n}=0.005,0.02,0.045,0.08,0.125,0.18 \mathrm{eV}$ для случая, когда энергия Ферми расположена между уровнями $E_{3}=0.045 \mathrm{eV}$, и $E_{4}=0.08 \mathrm{eV} \quad(N=3)$. При численном решении уравнения (4) ограничились максимальным количеством минизон $M=5$.
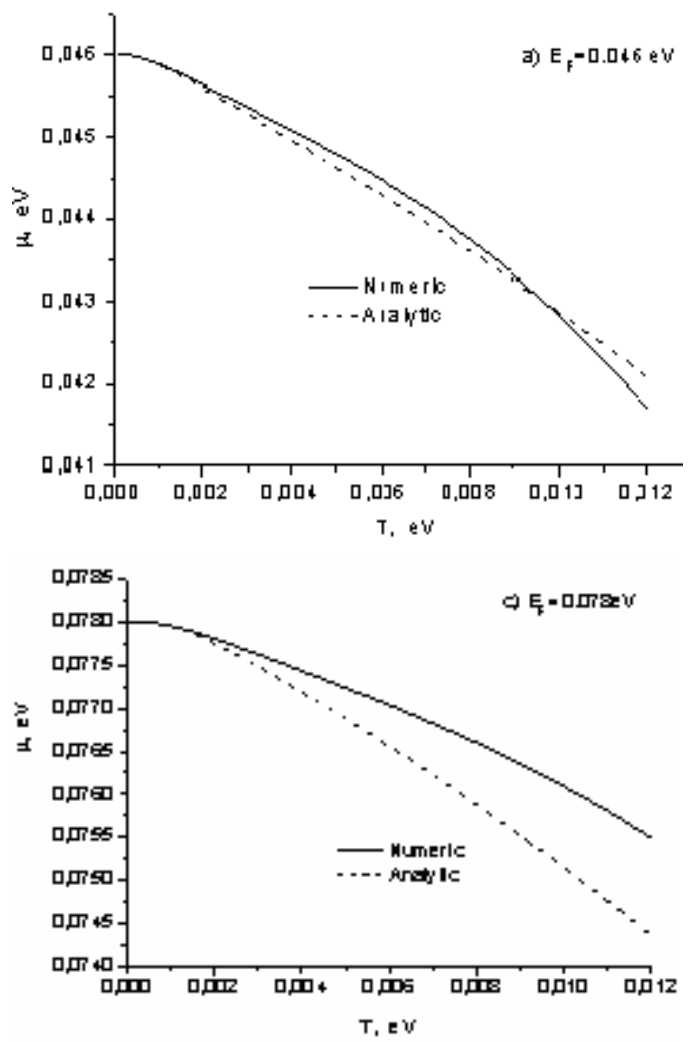

96

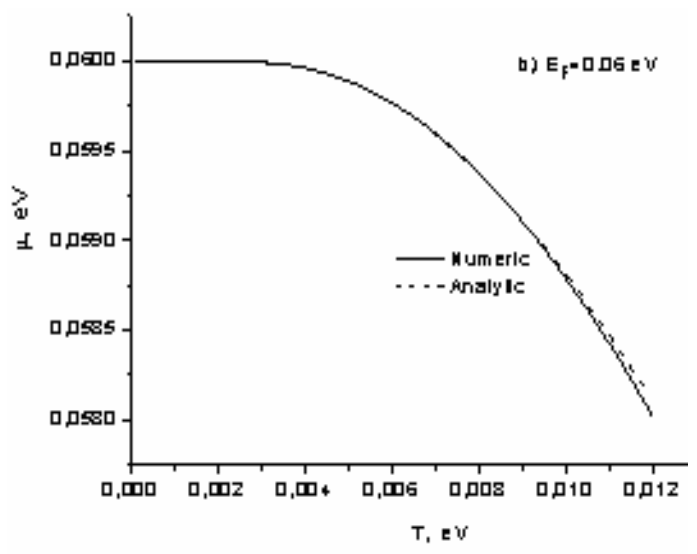

Рис. 1. Сравнение зависимостей $\mu(T)$, полученных по аналитической формуле (9), с результатами численного решения уравнения (4). 
Из этих графических зависимостей можно заключить, что если энергия Ферми $E_{F}$ расположена далеко от уровня размерного квантования (в данном случае от $E_{3}$ и $\left.E_{4}\right)$, то отчетливо наблюдается экспоненциально медленное уменьшение химического потенциала в широком интервале температур $0<T<0.01 \mathrm{eV}$, рис. $1 \mathrm{~b})$. Этот диапазон уменьшается по мере приближения энергии Ферми $E_{\mathrm{F}}$ к краям дна минизон $E_{3}$ и $E_{4}$ (рис. 1 а и с) соответственно.

На рис. 2 представлены сравнения зависимостей $\mu(T)$, полученных по аналитической формуле (13), с результатами численного решения уравнения (4) для разных значений энергии Ферми. При расчетах использованы следующие параметры спектра: $E_{1}=0.005 \mathrm{eV}, E_{n}=0.005,0.02,0.045,0.08,0.125,0.18 \mathrm{eV}$, для случая, когда энергия Ферми расположена между уровнями $E_{3}=0.045 \mathrm{eV}$, и $E_{4}=$ $0.08 \mathrm{eV}(N=3)$, но близко к уровню $E_{3}$. При численном решении уравнения (4) ограничились максимальном количеством минизон $M=5$.

Когда энергия Ферми достаточно близка к третьему уровню: $E_{F}=0.0458 \mathrm{eV}$ и $E_{3}=$ $0.045 \mathrm{eV}$, то, как видно из рис. 2a, аналитическая формула (13) и численная модель имеют общую точку пересечения $T_{3}=0.00346 \mathrm{eV}$. При удалении энергии Ферми от уровня $E_{3}: E_{F}=0.0484 \mathrm{eV}$ и $E_{3}=$ $0.045 \mathrm{eV}$ (рис. 2b), точка пересечения, полученная по аналитической формуле (13), равна $T_{3}=0.01502 \mathrm{eV}$, в то же время точный численный результат равен $T_{3}=0.0117 \mathrm{eV}$.

Из этих графических зависимостей можно заключить, что чем ближе энергия Ферми $E_{F}$ к уровню $E_{3}$, тем лучше выполняется линейная зависимость (13) и тем точнее оценка $T_{N}$ по формуле (16).

\section{V. ЗАКЛЮЧЕНИЕ}

Определение закона изменения химического потенциала с температурой является важной задачей для понимания термо-
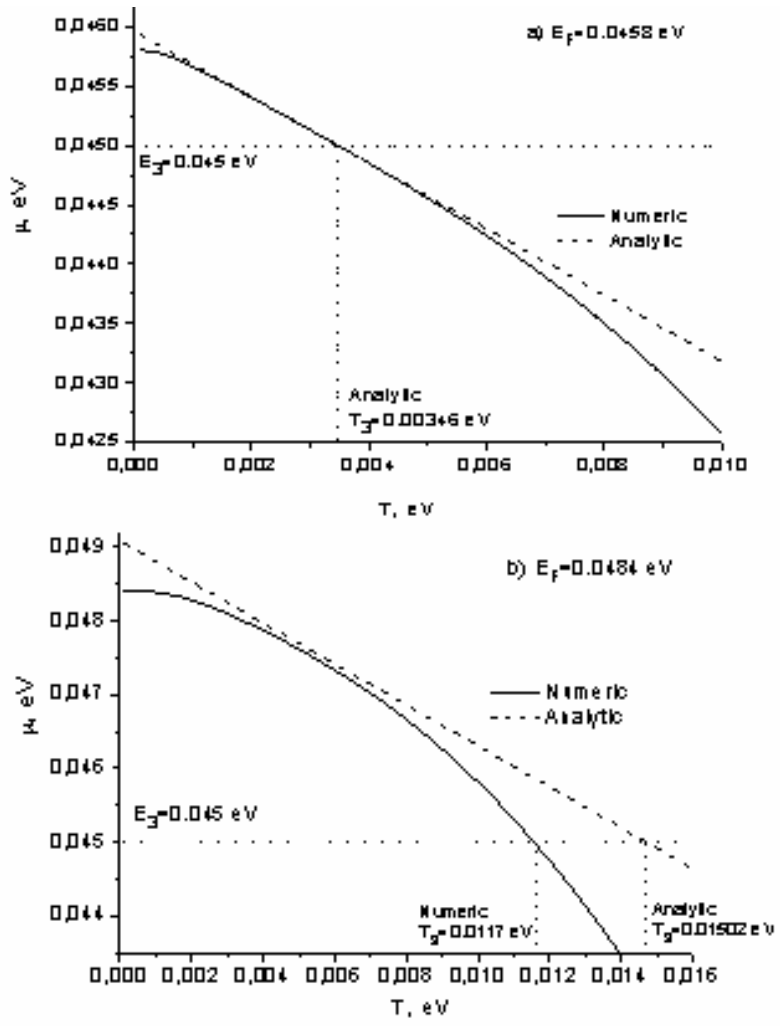

Рис. 2. Сравнение зависимостей $\mu(T)$, полученных по аналитической формуле (13), с результатами численного решения уравнения (4). динамических свойств любого газа, так как практически все наблюдаемые термодинамические параметры и кинетические коэффициенты определяются по этой зависимости.

Аналитические формулы для температурной зависимости химического потенциала квазидвумерного электронного (или дырочного) газа получены в приближении низких температур. Рассмотрены случаи, когда энергия Ферми 
расположена между уровнями размерного квантования и близка к этим уровням. Проанализирован также случай точного резонанса. Результаты показывают, что с ростом энергии Ферми зависимость химического потенциала от температуры меняется от линейного закона (в точках резонанса) к экспоненциально малому закону изменения (вдали от точки резонансов).

Поскольку темп изменения химического потенциала в точках резонанса максимален, то отсюда вытекает, что в этих точках тепловые переходы электронов на лежащие выше энергетические состояния более интенсивны. Это обусловлено резким скачком плотности энергетических состояний в точках резонанса. Из линейной зависимости (13) следует также закон квантования одночастичной энтропии $s(N)[12,18]$. Действительно, согласно (13)

$$
-(d \mu / d T)_{E_{F}}=\ln (2) /(N-1 / 2)=s .
$$

В работе использована модель идеального газа с простым параболическим спектром в одиночной квантовой яме. Таким образом, не учтен ряд факторов, такие как процессы столкновения, тепловой переход электронов из квантовой ямы на локальные уровни и зону проводимости широкозонного материала, а также влияние валентной зоны. Эти задачи требуют отдельного рассмотрения.

\section{ЛИТЕРАТУРА}

1. T. Ando, A.B. Fowler, and F. Stern, Reviews of Modern Physics 54, No.2, (1982).

2. G. Bastard. Wave Mechanics Applied to Semiconductor Heterostructures (Les Editions de Physique, 1988).

3. G. Bastard, J.A. Brunt, R. Ferreira. Electronic States in Sermiconductor Heterostructures (Acad. Press, 1991).

4. C.W.J. Beenaker, H. von Houten, Solid State Phys. (Acad. Press. Inc.) 44, (1991).

5. Physics of Low Dimensional Structures. Ed. by P. Butcher, N.H. March, M.P. Tosi (New York, Plenum Press, 1993).

6. S. Datta. Electronic Transport in Mesoscopic Systems (Cambridge Univ. Press, 1995).

7. Y. Imry. Introduction to Mesoscopic Physics (Oxford Univ. Press, 1997).

8. А.Я. Шик, Л.Г. Бакуева, С.Ф. Мусихин, С.А. Рыков. Физика низкоразмерных систем. Под ред. А.Я. Шика (СПб, Наука, 2001).

9. В.М. Гохфельд, Физика низких температур 31, $769-773$ (2005).

10. Н.В. Глейзер, А.М. Ермолаев, Г.И. Рашба, М.А. Соляник, Вісник ХНУ № 962, серія «Фізика», вип. 15, 15-20 (2011).

11. A.Yu. Kuntsevich, I.V. Tupikov, et al., Nat. Commun. 6, 7298 (2015).

12. A.A. Varlamov, A.V. Kavokin, and Y.M. Galperin, Phys.Rev. B 93, 155404 (2016); doi: 10.1103/PhysRevB.93.155404

13. И.В. Кукушкин, С.В. Мешков, В.Ф. Тимофеев, УФН 155, вып. 2, 219-264 (1988).

14. A.C.A. Ramos, G.A. Farias, N.S. Almeida, Phys. E: Low-Dimensional Systems and Nanostructures 43, 1878-1881 (2011).

15. R.J. Warburton, C. Gauer, A. Wixforth, J.P. Kotthaus, B. Brar, and H. Kroemer, Phys. Rev. B 53, 7903 (1996); http://dx.doi.org/10.1103/PhysRevB.53.7903

16. В.Я. Алешкин, В.И. Гавриленко, А.В. Иконников, Ю.Г. Садофьев, J.Р. Bird, S.R. Johnson, Y.-H. Zhang, ФТП 39, 71 (2005).

17. P.J. Baymatov, B.T. Abdulazizov, Ukr. J. Phys. 62, No. 1, $46-50$ (2017); doi: 10.15407/ujpe62.01.0046

18. P.J. Baymatov, A.G. Gulyamov, and B.T. Abdulazizov, Advances in Condensed Matter Physics, Hindawi Publishing Corporation, 2019, ID 8317278, https://doi.org/10.1155/2019/8317278 